\title{
Extramedullary Plasma Cell Neoplasms with Amyloidosis of the Head and Neck Region: A Series of two Rare Cases and Literature Review
}

\author{
Nupur Nerurkar ${ }^{1}$, Sohil Gala ${ }^{2}$, Ruchi Shah $^{3}$, Udbhav Mittal $^{4}$
}

\begin{abstract}
Extramedullary plasmacytomas (EMP) are rare malignant plasma cell neoplasms (PCNs) mainly affecting the head and the neck region. These are more commonly seen in adult males. These have variable outcomes and may even coexist with, or progress to multiple myelomas (MMs). Hence, a systemic examination is a must, to rule out generalized involvement. The presence of monoclonal plasma cells on histopathology explains the relationship of PCNs with amyloidosis. Treatment may be surgical for accessible tumors or low to moderate dose radiation as indicated. Long-term surveillance is a must. We discuss two cases of extramedullary PCNs with amyloidosis involving the head-neck region. Both the patients underwent surgical excision followed by radiation for the residual disease and recurrence prevention. Systemic involvement was ruled out and the patients have been advised regular follow-up.

Keywords: Extramedullary plasmacytomas, Plasma cell Neoplasms, Plasmacytoma with amyloidosis.

International Journal of Phonosurgery and Laryngology (2018): 10.5005/jp-journals-10023-1163
\end{abstract}

\section{INTRODUCTION}

Plasma cell neoplasms (PCNs) comprise of three clinical entities namely MMs, solitary bone plasmacytoma (SBP), and Extramedullary plasmacytomas (EMPs). ${ }^{1}$

EMPs consist of only $3 \%$ of all plasma cell tumors. ${ }^{1,2}$ These tumors can be found anywhere in the body, but over $80 \%$ are found in the head and the neck region. ${ }^{1,3}$ These are more common in men and typically seen in the 6 th-8th decade of life. ${ }^{1,2}$

Rarely, EMPs may be associated with amyloid deposits. ${ }^{4}$ One of the most common forms of amyloid protein is light chain protein which is derived from plasma cells and, hence, there is a pathogenic relationship between PCNs and amyloid. ${ }^{4,5}$

Although slow growing and localized EMPs can be closely associated with, or may progress to $\mathrm{MM}$, therefore, an early diagnosis and regular follow-up are essential. Treatment options are surgery and/or radiation either singly or in combination.

We report two rare cases of extramedullary PCNs along with amyloid deposits involving the head and the neck region.

\section{CASE I}

A 70-year-old Indian male presented with long standing dry cough and throat irritation. The patient gave a history of similar complaints 6 years back for which he was treated, but details are not available. A flexible naso-laryngo-pharyngoscopic examination revealed the presence of two smooth sessile well-defined lesions, one arising from the nasopharngeal surface of the soft palate on the left side and the other from the left lateral nasopharyngeal wall (Fig. 1). These lesions occupied the left side of chona and nasopharyngeal lumen without causing any significant airway compromise which explained the absence of nasal symptoms.

A contrast CT (Fig. 2) revealed moderately enhancing, welldefined soft tissue lesion in the left lateral pharyngeal wall, in close proximity to the tonsillo-lingual sulcus and soft palate.

We scheduled the patient for an excisional biopsy and intraoperative frozen section examination with subsequent decision making on table. The lesions were excised using a combined per
${ }^{1-4}$ Voice Clinic, Bombay Hospital and Research Centre, Mumbai, Maharashtra, India

Corresponding Author: Sohil Gala, Voice Clinic, Bombay Hospital and Research Centre, Mumbai, Maharashtra, India, Phone: +91 223531018, e-mail: sohilgala17@gmail.com

How to cite this article: Nerurkar N, Gala S, et al. Extramedullary Plasma Cell Neoplasms with Amyloidosis of the Head and Neck Region: A Series of two Rare Cases and Literature Review. Int J Phonosurg Laryngol 2018;8(2):79-82.

Source of support: Nil

Conflict of interest: None

oral and endoscopic endonasal approach. The frozen section results revealed a PCN. All accessible tumor was excised leaving behind part of the lesion deep into the lateral nasopharyngeal wall in close proximity to the eustachian tube. The final histopathology revealed a plasmacytoma with amyloid deposits with immunohistochemistry (IHC) positive for CD-138 and MUM-1 and plasma cells were $\lambda$ light chain restricted.
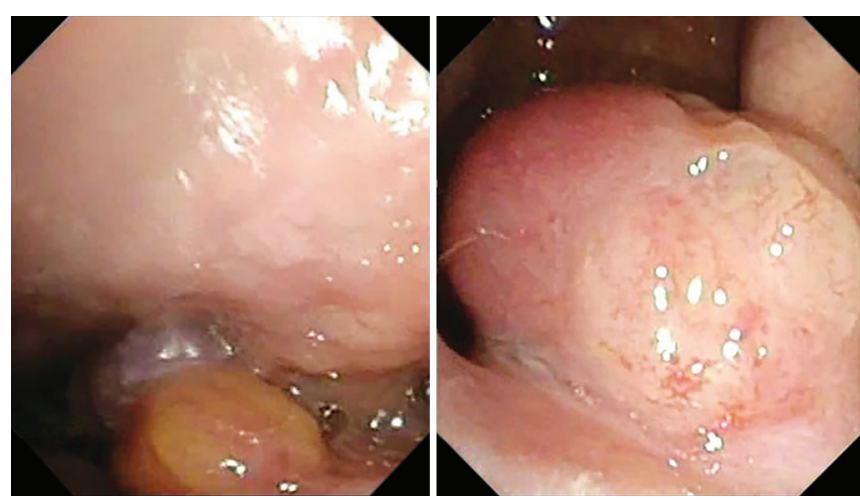

Fig. 1: Flexible nasal endoscopy showing well-defined, sessile globular mass arising from the nasopharyngeal surface of the soft palate and the left lateral nasopharyngeral wall 

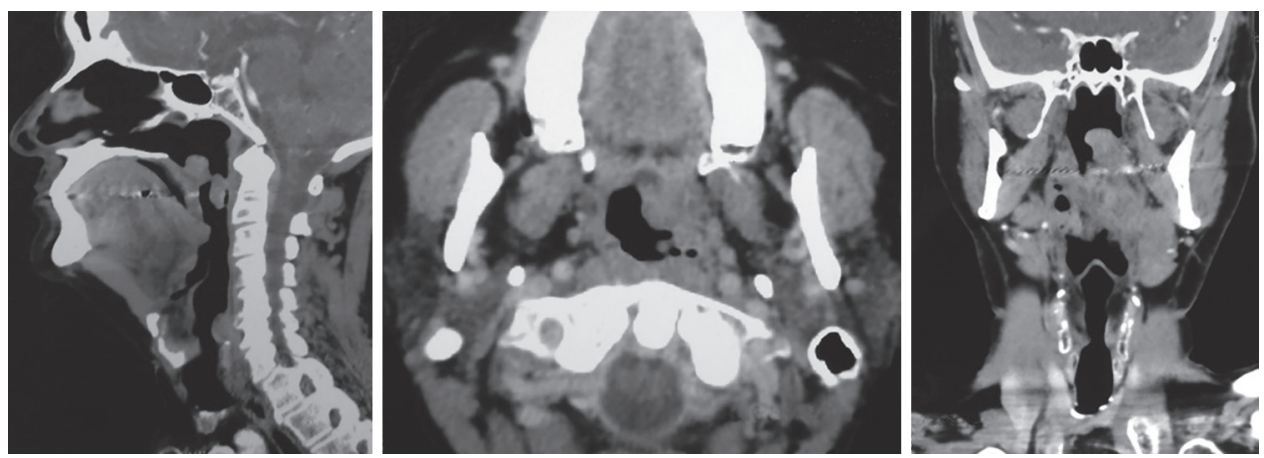

Fig. 2: Para-sagittal, axial, and coronal view of contrast-enhanced CT showing the lesions from the soft palate and the pharyngeal wall

Bone marrow biopsy, hematological investigation, plasma/urine electrophoresis, and skeletal survey were unremarkable. Whole body positron emission tomography-computed tomography (PET$\mathrm{CT}$ ) had evidence of local residual disease with no other systemic lesion. A conclusive diagnosis of EMP with amyloid deposits was made.

As per the hematologists advice, our patient was referred for low-dose postoperative radiation in view of residual local disease. He has been advised a close surveillance with us and the hematologists for a minimum period of 5 years and if possible life long and is currently asymptomatic.

\section{CAse II}

A 63-year-old male presented with a history of hoarseness and dry cough, about 6 years back to our clinic. He was diagnosed with ventricular phonation and laryngo-pharyngeal reflux on video stroboscopic examination. He was advised voice therapy and medical management for the above findings and had some amount of improvement but did not show complete resolution of symptoms.

The patient reported worsening of symptoms over the last few months, for which he visited our clinic. The stroboscopic examination revealed a well-defined lesion over the superior surface of right true vocal fold with some amount of ventricular phonation (Fig. 3).

We scheduled our patient for an excisional biopsy of the lesion with frozen section which revealed amyloid deposits. The final histopathological diagnosis of the lesion was a PCNs with amyloidosis. IHC showed monoclonal plasma cells with $\lambda$ light chain restriction.

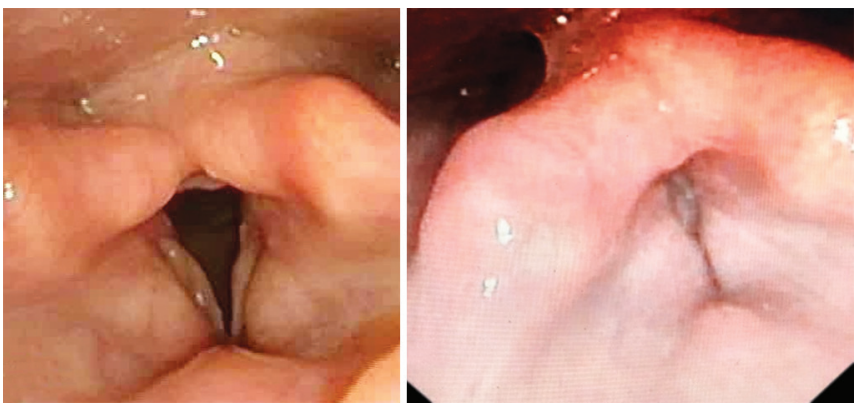

Fig. 3: Stroboscopic image showing both true vocal folds in full abduction with smooth lesion over the superior surface of right true fold and ventricular phonation pattern on adduction
The patient was referred to a hematologist for further management. Hematological investigations, electrophoresis, bone marrow biopsy, echocardiography, and a full-body positron emission tomography (PET) CT scan were unremarkable, thus, ruling out systemic plasma cell dyscrasias and amyloidosis. The patient was referred to the National Amyloidosis Centre, United Kingdom for a serum amyloid protein (SAP) scintigraphy scan which did not show any visceral amyloid deposits.

A contrast CT neck was advised which revealed evidence of soft tissue infiltration in the right false vocal fold abutting the superior surface of the right true vocal folds, with intact thyroid cartilage (Fig. 4).

In view of the radiographically detected lesion and monoclonal plasma cell population on IHC, the patient was referred for postoperative radiation therapy to the larynx. The patient is under close surveillance and has been advised regular follow-up.

\section{Discussion}

EMPs are rare soft tissue malignant neoplasm accounting for less than $1-2 \%$ of total malignancies with an occurrence of $3.5 / 100000$ per year. ${ }^{6-8}$

EMPs belong to the family of PCNs which also include MM and SBP. EMPs account for only about $3 \%$ of total PCNs. EMPs differ from MM mainly in the fact that there is no plasma cell infiltration in bone marrow biopsy, and no systemic involvement. ${ }^{6,9}$ Histopathologically both have identical features and EMP can only be diagnosed once MM is ruled out.

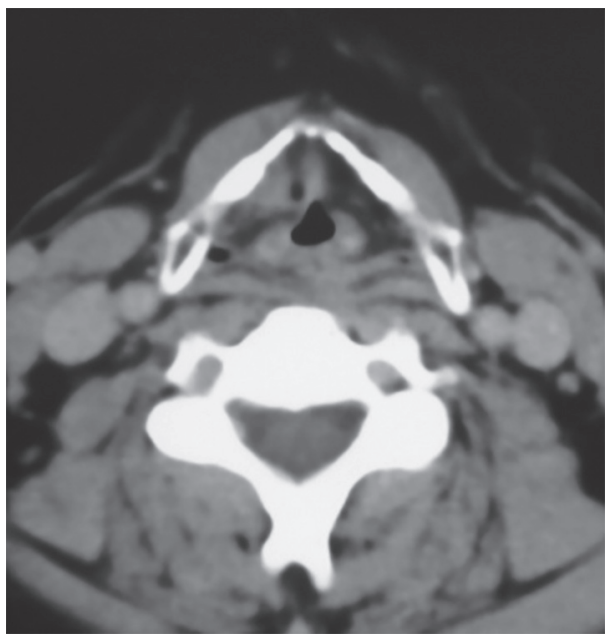

Fig. 4: Axial section CT neck showing soft tissue density occupying the right false vocal folds with intact thyroid cartilage lamina 
Over $80 \%$ of EMPs arise from the head and the neck region mainly involving the nose, paranasal sinuses, nasopharynx, oropharynx, and larynx in the order of decreasing frequency. ${ }^{1,10}$ EMPs are about 4 times more common in males and occur in the 6th-8th decades of life. ${ }^{2}$

Our first patient was a 70-year-old male and the lesion involved the soft palate and the lateral nasopharyngeal wall. The second patient was a 63-year-old male with the involvement of the right true and false vocal folds, and the ventricle. Small lesions of the nasopharynx, not causing significant luminal compromise, will be asymptomatic usually and, hence, present very late in the course of disease. On the contrary, a patient with a laryngeal involvement will have early clinical symptoms like dyspnea and/ or dysphonia.

Radiological studies are important to determine the site of origin, extent, subsites involved, vascularity, rule out differentials, and lastly for surveillance and treatment monitoring.

Plasmacytoma can be confirmed only on histopathology and IHC. Histopathology typically shows connective tissue infiltration by plasma cells organized as sheets, islands, or nodules with different degrees of differentiation. ${ }^{11}$ Differentials like inflammatory plasma cell infiltration, lymphoplasmacytoid lymphoma, and plasma cell granuloma can be ruled out with $\mathrm{IHC}$. IHC can also determine the nature of the immunoglobulin light chain ( $k$ or $\lambda) .{ }^{12}$ CD138 and CD38 are specific markers while $C D 45, \mathrm{BCL}-2$, and $\mathrm{EMA}$ are nonspecific to EMP, which differentiates from a carcinoma which stains for $C K$ and CD40. CD56 is expressed in 70\% of the cases of MM as compared to $10 \%$ of EMP. ${ }^{13,14}$ The presence of $k$ or $\lambda$ light chains population in plasmacytoma suggests a relationship with amyloidosis (AL form).

Our first patient was positive for CD-138 and MUM-1 and the cells were $\lambda$ light chain restricted. There was the presence of congophilic amyloid deposits with apple green birefringence with polarized light (Figs 5A and B).

The second patient showed plasma cell infiltration along with stroma of acellular, homogenous, eosinophilic material which was congo red positive on histopathology (Fig. 5C). The IHC confirmed the plasma cell infiltrate having monoclonal lineage with $\lambda$ chain restriction.

In a study by Krishnan et al., only 1 one out of 14 patients with amyloidosis had coexistent plasmacytoma. ${ }^{15}$ Pambuccian et al. reported two cases of amyloidosis with plasmacytoma. ${ }^{16}$ Eilam et al. reported a case of plasmacytoma of the nasal cavity that involved the palate, ethmoidal, and maxillary sinuses and contained deposits of amyloid. ${ }^{17}$ Ustün et al. reported a case of extramedullary plasmacytoma of the parotid gland with extensive amyloid deposition. ${ }^{18}$ Majority of these cases had systemic involvement in the form of MMs and isolated plasmacytoma with amyloid deposits have been rarely reported. We have encountered nine cases of localized amyloidosis of the larynx in our experience, of which, only one case has coexisting PCN on histopathology.

Once diagnosed as a plasmacytoma, a systemic examination is mandatory. Complete hemogram, calcium profile, serum and urine electrophoresis, quantitative immunoglobulin detection, skeletal survey, bone marrow biopsy, and a full-body PET CT scan are indicated to rule out systemic involvement or a MM. ${ }^{19,20}$

A recent technique called as a SAP scintigraphy scan shows the distribution and amount of amyloid in the organs throughout the body without the need for biopsies, which can only sample a microscopic amount of tissue from a limited number of places. SAP scanning has revolutionized the understanding of the natural course of amyloidosis and its response to treatment. Unfortunately, the method is complex; requires sophisticated materials, technology, and equipment; and is very expensive. ${ }^{21}$ Our second patient underwent this SAP scintigraphy scan at the National Amyloidosis Centre, United Kingdom, which did not show any visceral amyloid deposits and, thus, systemic amyloidosis was ruled out.

If the systemic examination as described above is normal, a confirmatory diagnosis of EMP can be made as per these criteria (1-4 mandatory): $:^{12}$

1. Histological evidence of plasmacytoma

2. Bone marrow aspiration showing less than $10 \%$ atypical plasma cells

3. Absence of clinical and radiological evidence of skeletal lesions

4. Unchanged levels of serum proteins or electrophoresis of serum/ urinary proteins

5. Absence of anemia ( $80-85 \%$ patients with MMs have normochromic normocytic anemia)

EMPs once diagnosed are known to have variable outcomes. They may remain localized, may cause aggressive local extension to adjoining bones and soft tissues, may disseminate to lymphatics, and may even progress to MM. Wiltshaw stated in his study that $40 \%$ of extramedullary plasmacytomas spread beyond the primary site and/or underwent lymphatic drainage. Of these, $62 \%$ of patients had deposits in soft tissues and visceral organs, and $81 \%$ developed bone lesions. An extramedullary plasmacytoma at this stage is called disseminated extramedullary plasmacytoma, and its prognosis is undoubtedly better than that of MMs. ${ }^{22}$ However, the possibility for conversion of MMs exists. Its incidence rate
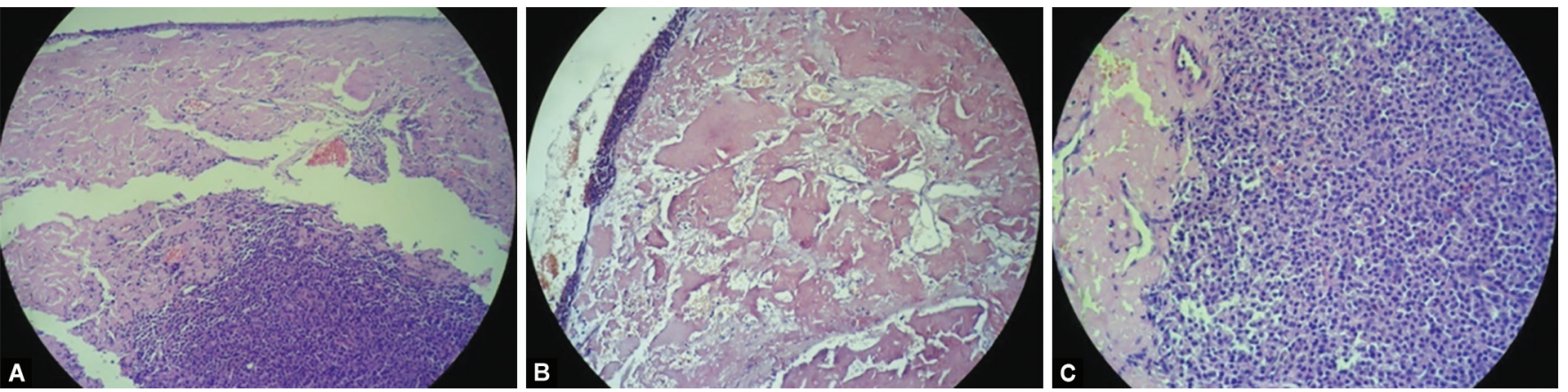

Figs 5 A to C: Postoperative histopathology showing; (A) The presence of plasmacytoma with amyloid deposits; (B) Amyloid confirmed on Congo Red staining; (C) Extensive amyloid deposits with PCN 
varies from 15 to $20 \%$ and its prognosis is poor. ${ }^{12}$ Also the rate of conversion is higher if underlying bone is involved. ${ }^{1}$ Hence, there is a need for regular and aggressive surveillance.

Treatment approaches include surgery, radiation, chemotherapy either singly or in combinations. ${ }^{19}$ Surgery is indicated first for a histopathology in the form of punch, incisional, or excisional biopsy. Also it is the preferred treatment for localized and accessible lesions which can be completely excised without causing significant postoperative morbidity. EMPs are strongly radiosensitive tumors. Radiation is treatment of choice if lesion is not localized or accessible, incomplete excision or residual, and in cases of disseminated disease. Some may even propose radiation as the primary treatment modality in all cases. Typically, a low to medium dose radiation (40-46 Gy) is employed with good results and minimal toxicity. ${ }^{1}$ Chemotherapy may be added for disseminated and advanced disease. ${ }^{12}$

The entire surgically accessible tumor was excised in both our cases with postoperative radiation for residual disease and to reduce chances of recurrence.

All cases of EMPs of the head and the neck region justify regular and a long-term follow-up with the otorhinolaryngologists and hematologists owing to the unpredictable outcomes. Monitoring of all cases for a period of up to 5 years is warranted in most cases.

\section{Conclusion}

EMPs are rare and aggressive PCNs which most commonly occur in the head and the neck region and, hence, must be kept in mind by all otorhinolaryngologists as a differential for unusual clinical presentations. Owing to the presence of monoclonal plasma cells, PCNs share a pathogenic relationship with amyloidosis. Once diagnosed, a multidisciplinary approach is essential to rule out any systemic involvement. These have unpredictable outcomes and hence a long term monitoring is a must. Progression to MM is an indicator of poor prognosis.

\section{References}

1. Gholizadeh N, Mehdipour M, et al. Extramedullary Plasmacytoma of the Oral Cavity in a Young Man: A Case Report. J Dent Shiraz Univ Med Sci 2016 June;17(2):155-158.

2. Majumdar S, Raghavan U, et al. Solitary plasmacytoma and extramedullary plasmacytoma of the paranasal sinuses and soft palate. J Laryngol Otol 2002;116:962-965. DOI: 10.1258/00222150260369561.

3. Anil S. Solitary plasmacytoma of the maxilla-a case report and review of the literature. Gen Dent 2007;55:39-43.

4. Sharma N, Sharma S, et al. Plasmacytoma with amyloidosis masquerding as tuberculosis on cytology. J Cytol 2009 Oct-Dec; 26(4):161-163. DOI: 10.4103/0970-9371.62190.
5. Nagasaka T, Lai R, et al. Localized amyloidosis and extramedullary plasmacytoma involving the larynx of a child. Hum Pathol 2003;32:132-134. DOI: 10.1053/hupa.2001.20896.

6. Kwon D, Myoung H. Case Report: Extramedullary Plasmacytoma of Soft Palate. Korean J Oral Maxillofac Pathol 2016;40(3):832-838. DOI: 10.17779/KAOMP.2016.40.3.005.

7. Kapadia SB, Desai U, et al. Extramedullary Plasmacytoma of the Head and Neck: A Clinicopathologic Study of 20 Cases. Medicine (Baltimore) 1982;61:317-329. DOI: 10.1097/00005792-198209000-00004.

8. Mendenhall WM, Charles MM, et al. Solitary plasmacytoma of bone and soft tissues. Am J Otolaryngol 2003;24:395-399. DOI: 10.1016/ S0196-0709(03)00092-9.

9. Neville BW, Damm DD, et al. Oral and maxillofacial pathology. Philadelphia Saunders 2008;606-607.

10. Rawat DS, Grover M, et al. Extramedullary Plasmacytoma of Nasal Cavity: A Rare Entity. Clinical Rhinology: An International Journal 2010;3:39-41. DOI: 10.5005/jp-journals-10013-1025.

11. Yoshimura $Y$, Takada K, et al. Two cases of plasmacytoma in the oral cavity. Int J Oral Surg 1976 Apr;5(2):82-91.

12. dos Anjos Corvo MA, Granato L, et al. Extramedullary nasal plasmacytoma: literature review and a rare case report. Int Arch Otorhinolaryngol 2013;17(2):213-217. DOI: 10.7162/S180997772013000200016.

13. Dalal B S, Sarkar P, et al. Soft-tissue amyloidoma with associated plasmacytoma. Indian Journal of Pathology and Microbiology Oct-Dec 2016;59(4):535-537. DOI: 10.4103/0377-4929.191821.

14. Zuo Z, Tang Y, et al. Ex traosseous (extramedullary) plasmacytomas: a clinicopathologic and immunophenotypic study of 32 Chinese cases. Diagn Pathol 2011;6:123. DOI: 10.1186/1746-1596-6-123.

15. Krishnan J, Chu WS, et al. Tumoral presentation of amyloidosis (amyloidomas) in soft tissues: a report of 14 cases. Am J Clin Pathol 1993;100:135-144. DOI: 10.1093/ajcp/100.2.135.

16. Pambuccian SE, Horyd ID, et al. Amyloidoma of bone, a plasma cell/ plasmacytoid neoplasm: report of three cases and review of the literature. Am J Surg Pathol 1997;21:179-186. DOI: 10.1097/00000478199702000-00007.

17. Eilam O, Liepnieks JJ, et al. Plasmacytoma of the nasal cavity with lambda III amyloid deposition. Amyloid 1995;2:31-35. DOI: 10.3109/13506129509031885.

18. Ustün MO, Ekinci N, et al. Extramedullary plasmacytoma of the parotid gland. report of a case with extensive amyloid deposition masking the cytologic and histopathologic picture. Acta Cytol 2001;45:449-453. DOI: 10.1159/000327648.

19. Huoh KC, Van Zante A, et al. Case Report: Extramedullary Plasmacytoma of the Tonsil. Case Rep Otolaryngol 2011;2011:430809. DOI: 10.1155/2011/430809.

20. Bachar $G$, Goldstein $D$, et al. Solitary extramedullary plasmacytoma of the head and neck-Long-term outcome analysis of 68 cases. Head and Neck 2008;30(no. 8):1012-1019. DOI: 10.1002/hed.20821.

21. Cytawa W, Jacek T, et al. Nuclear Imaging of Amyloidosis. Pol J Radiol 2014;79:222-227. DOI: 10.12659/PJR.890147.

22. Wiltshaw E. The natural history of extramedullary plasmocitoma and its relation to solitary mieloma of bone and myelomatosis. Medicine 1976;55:217-238. DOI: 10.1097/00005792-197605000-00002. 